\title{
Adesão ao tratamento farmacológico em pacientes com doenças inflamatórias intestinais: uma revisão integrativa da literatura
}

\author{
Adherence to pharmacological treatment in patients with inflammatory intestinal diseases: \\ an integrative literature review
}

\begin{abstract}
Adherencia al tratamiento farmacológico en pacientes con enfermedades intestinales inflamatorias: una revisión integrativa de la literatura
\end{abstract}

Fernanda Gomes de Souza ${ }^{1 *}$, Ana Cristina Lo Prete², Carolina Heitmann Mares Azevedo Ribeiro.

\section{RESUMO}

Objetivo: Conhecer e discutir por meio de levantamento bibliográfico sobre a adesão ao tratamento farmacológico em portadores de Doença Inflamatória Intestinal (DII). Métodos: A seleção dos estudos foi realizada por meio das bases de dados LILACS, MEDLINE e PubMED, por meio dos descritores: "doença inflamatória intestinal", "doença de crohn", "retocolite ulcerativa" e "Adesão" nos idiomas português, inglês ou espanhol. Resultados: Foram incluídos os artigos disponíveis de forma completa, com acesso gratuito e adequados ao tema proposto. Foram pré-selecionados vinte e oito artigos e destes, oito foram excluídos de acordo com os critérios estabelecidos. O método mais utilizado nos estudos para analisar adesão ao tratamento farmacológico nas DII foi o indireto. Entre os preditores de não adesão ao tratamento, destacamse fatores emocionais como a percepção negativa do paciente em relação a doença, menor idade ao diagnóstico, esquecimento de doses do medicamento, indisponibilidade de medicamentos, sentir-se melhor, efeitos adversos, custo do tratamento, status socioeconômico elevado, etilismo e tabagismo, dentre outros. Considerações finais: Independente dos métodos de adesão utilizado nos estudos analisados, foi observada a ocorrência de adesão inadequada em proporção considerável de pacientes com DII.

Palavras-chave: Doença de Crohn, Retocolite ulcerativa, Adesão ao tratamento.

\begin{abstract}
Objective: Discussing, through a bibliographic survey, about pharmacological treatment adherence in patients with Inflammatory Bowel Disease (IBD). Methods: The selection of studies was carried out using the LILACS, MEDLINE and PubMED databases, using the descriptors: "inflammatory bowel disease", "crohn's disease", "ulcerative colitis" and "Adhesion" in Portuguese, English or Spanish. Results: The articles available in full, with free access and appropriated to the proposed theme were included. Twenty-eight articles were preselected and of these, eight were excluded according to the established criteria. The most used method to analyze adherence to pharmacological treatment in IBDs was the indirect method. Among the predictors of non-adherence, emotional factors standout such as the patient's negative perception of the disease, younger age at diagnosis, forgetting medications, drug unavailability, feeling better, adverse effects, treatment cost, high socioeconomic status, alcoholism and smoking, among others. Final considerations: Regardless of the methods of adherence used in the studies analyzed, the occurrence of inadequate adherence was observed in a considerable proportion of patients with IBD.
\end{abstract}

Keywords: Crohn's disease, Ulcerative colitis, Treatment adherence.

1 Universidade Federal do Pará (UFPA), Belém - PA.

2Universidade São Judas Tadeu (USJT), São Paulo - SP. *E-mail: carolmheitmann@hotmail.com 


\section{RESUMEN}

Objetivo: Conocer y discutir a través de una encuesta bibliográfica sobre la adherencia al tratamiento farmacológico en pacientes con Enfermedad Inflamatoria Intestinal (EII). Métodos: La selección de los estudios se llevó a cabo utilizando las bases de datos LILACS, MEDLINE y PubMED, utilizando los descriptores: "enfermedad inflamatoria intestinal", "enfermedad de Crohn", "colitis ulcerosa" y "Adhesión" en portugués, inglés o español. Resultados: Se incluyeron los artículos disponibles en su totalidad, con acceso libre y apropiados para el tema propuesto. Veintiocho artículos fueron preseleccionados y de estos, ocho fueron excluidos de acuerdo con los criterios establecidos. El método más utilizado en los estudios para analizar la adherencia al tratamiento farmacológico en las Ell fue el indirecto. Entre los predictores de no adherencia al tratamiento destacan los factores emocionales, como percepción negativa de la enfermedad por parte del paciente, menor edad al momento del diagnóstico, omisión de dosis de medicación, indisponibilidad de medicación, sentirse mejor, efectos adversos, costo de tratamiento, alto nivel socioeconómico, alcoholismo y tabaquismo, entre otros. Consideraciones finales: Independientemente de los métodos de adherencia utilizados en los estudios analizados, se observó la aparición de adherencia inadecuada en una proporción considerable de pacientes con Ell.

Palabras clave: Enfermedad de Crohn, Colitis ulcerosa, Adherencia al tratamiento.

\section{INTRODUÇÃO}

A retocolite ulcerativa (RCU) e a doença de Crohn (DC) são as principais doenças que compõem as Doenças Inflamatórias Intestinais (DII). São enfermidades crônicas com ocorrência crescente em todo o mundo, caracterizadas por períodos de exacerbação e remissão (MOLODECKY NA, et al., 2012).

A DC e a RCU apresentam sintomas parecidos, porém possuem algumas características fisiopatológicas fazendo com que se diferenciem entre si (MARANHÃO DDA, et al., 2015). A RCU é restrita à mucosa e submucosa, com inflamações que envolvem a mucosa do cólon e reto, levando ao desenvolvimento de úlceras. A doença tem início no reto podendo se estender a todo o cólon de maneira contínua (BARBOSA JPC, 2018). Está associada a diversas manifestações extraintestinais, que podem acometer articulações, fígado e vias biliares, ossos, pele, olhos e cascata de coagulação. Artropatia inflamatória e colangite esclerosante primária são as manifestações extraintestinais mais descritas (CONRAD K, et al., 2014).

A DC se caracteriza por inflamação crônica, podendo atingir uma ou várias porções do intestino, principalmente íleo e cólon. Também está associada a manifestações extraintestinais, afetando principalmente as articulações e pele (BAUMGART DC e SANDBORN WJ,2012).

Mesmo com diversos estudos, a etiologia de ambas ainda não foi totalmente esclarecida. Todavia, acredita-se que as mesmas se originam de desordens imunológicas na microbiota, além de fatores ambientais, genéticos e estilo de vida que também podem estar relacionados ao aparecimento da doença (SOUZA ACG, et al., 2015).

As DII representam um problema de saúde pública em algumas regiões da América do Norte e da Europa ocidental, enquanto na Ásia ainda possui um número incipiente de casos (LEDDIN D, et al., 2014). Dados revelam que indivíduos caucasianos, habitantes de áreas urbanas e industriais são mais acometidos por doenças de caráter autoimune, incluindo neste contexto pacientes com DII, porém têm-se observado novos casos em áreas que antes possuíam baixa prevalência, concomitante ao processo de desenvolvimento e urbanização (LOGAN I e BOWLUS CL, 2010).

No Brasil, os dados de incidência e prevalência são restritos, pois as DII não são de notificação obrigatória, há deficiência de registros de serviços públicos e falta de registros do atendimento da rede privada. No entanto, alguns estudos têm apontado para um aumento das DII no Brasil, entre eles o estudo epidemiológico de Parente JM, et al. (2015) realizado em um centro de referência de DIl no estado do Piauí, na região Nordeste brasileira, que identificou índice anual de ocorrência de DIl da região de 0,08 casos/100.000 habitantes/ano, em 1988, para 1.53 casos/100.000 habitantes/ano, em 2007, e 12,8 casos /100.000 habitantes/ano em 2012. 
Outros estudos epidemiológicos, como os de Silva BC, et al. (2015) e Gasparini RG (2018), mostram claro aumento da casuística de casos ambulatoriais e hospitalares de DII. Um estudo epidemiológico recente sobre a prevalência das DII no Brasil identificou 162.894 pacientes com DII. A distribuição da prevalência demonstrou um gradiente Norte-Sul, com prevalência maior ao Sul-sudeste do país (MOREIRA AL, et al., 2019).

Estas doenças possuem tratamento medicamentoso prolongado e, muitas vezes, por tempo indeterminado. Desse modo, para maior eficácia da terapêutica, é essencial haver adesão do paciente ao regime medicamentoso prescrito (MISZPUTEN SJ, 2011; CARDOZO WS e SOBRADO CW, 2015).

A adesão pode ser conceituada como o grau de concordância entre o comportamento de uma pessoa em relação às orientações do médico ou de outro profissional de saúde e pode ser mensurada por meio de métodos diretos e indiretos (WHO, 2003; OBRELI-NETO PR, et al., 2012).

O primeiro baseia-se em detecção do fármaco ou metabólito em fluidos biológicos pela adição de um marcador e observação direta do paciente. Já o indireto é fundamentado em informações fornecidas pelo paciente ou outras estimativas indiretas, as quais se correlacionam com o comportamento de adesão do paciente, como por exemplo: entrevista ao paciente, questionários estruturados, contagem manual de comprimidos, registro de retirada de medicamento em farmácias ou sistemas de monitoramento de eventos de medicação (OBRELI-NETO PR, et al., 2012).

O baixo grau de adesão pode afetar negativamente a evolução clínica do paciente e sua qualidade de vida, sendo considerado um problema relevante que pode trazer consequências pessoais, sociais e econômicas (SOUZA TT, 2013; GLOMBIEWSKI JA, et al., 2012). No que diz respeito especificamente à não adesão farmacológica dos pacientes com DII, esta é associada ao aumento da atividade da doença, às recaídas, à maior morbimortalidade, ao aumento do gasto em saúde, à má qualidade de vida e à maior incapacitação (HANAUER SB e PRESENT DH, 2003).

Desse modo, considerando a relevância da adesão ao tratamento medicamentoso nas DII, nesta revisão foram revisados os dados de adesão, os preditores de não adesão e as estratégias de intervenção para melhorar a adesão aos medicamentos.

\section{MÉTODOS}

O trabalho trata-se de uma revisão integrativa sobre a adesão ao tratamento medicamentoso em indivíduos com DII. A seleção dos estudos foi realizada nas bases de dados Literatura Latino-americana e do Caribe em Ciências da Saúde (LILACS), Sistema Online de Busca e Análise de Literatura Médica (MEDLINE) e base de dados bibliográficos da Biblioteca Nacional de Medicina dos Estados Unidos da América (US National Library of Medicine (NLM) na interface PubMed), por meio dos descritores: "doença inflamatória intestinal", "doença de Crohn", "retocolite ulcerativa" e "Adesão" nos idiomas português, inglês ou espanhol.

Os critérios de inclusão definidos para a seleção dos artigos foram: artigos que apresentavam texto completo disponível nos idiomas português, inglês ou espanhol, adequação ao tema proposto, com livre acesso e publicados nos últimos quatro anos. Foram excluídos artigos de revisão e estudos em pacientes pediátricos, estes últimos pelas particularidades que envolvem a ocorrência de DII nesse grupo de pacientes.

Para a delimitação da amostra, primeiramente foi realizada a identificação dos artigos conforme critérios de inclusão, seguido por triagem baseada na leitura dos títulos e resumos, com posterior eliminação dos artigos que não se enquadrassem na temática da revisão ou que estivessem duplicados nas bases de dados.

Posteriormente, foi feita a análise dos artigos elegíveis com base na leitura integral de seu conteúdo, excluindo-se os artigos não adequados para essa revisão, com posterior seleção final da amostra (Figura 1). 
Figura 1 - Fluxograma das etapas de seleção dos artigos.

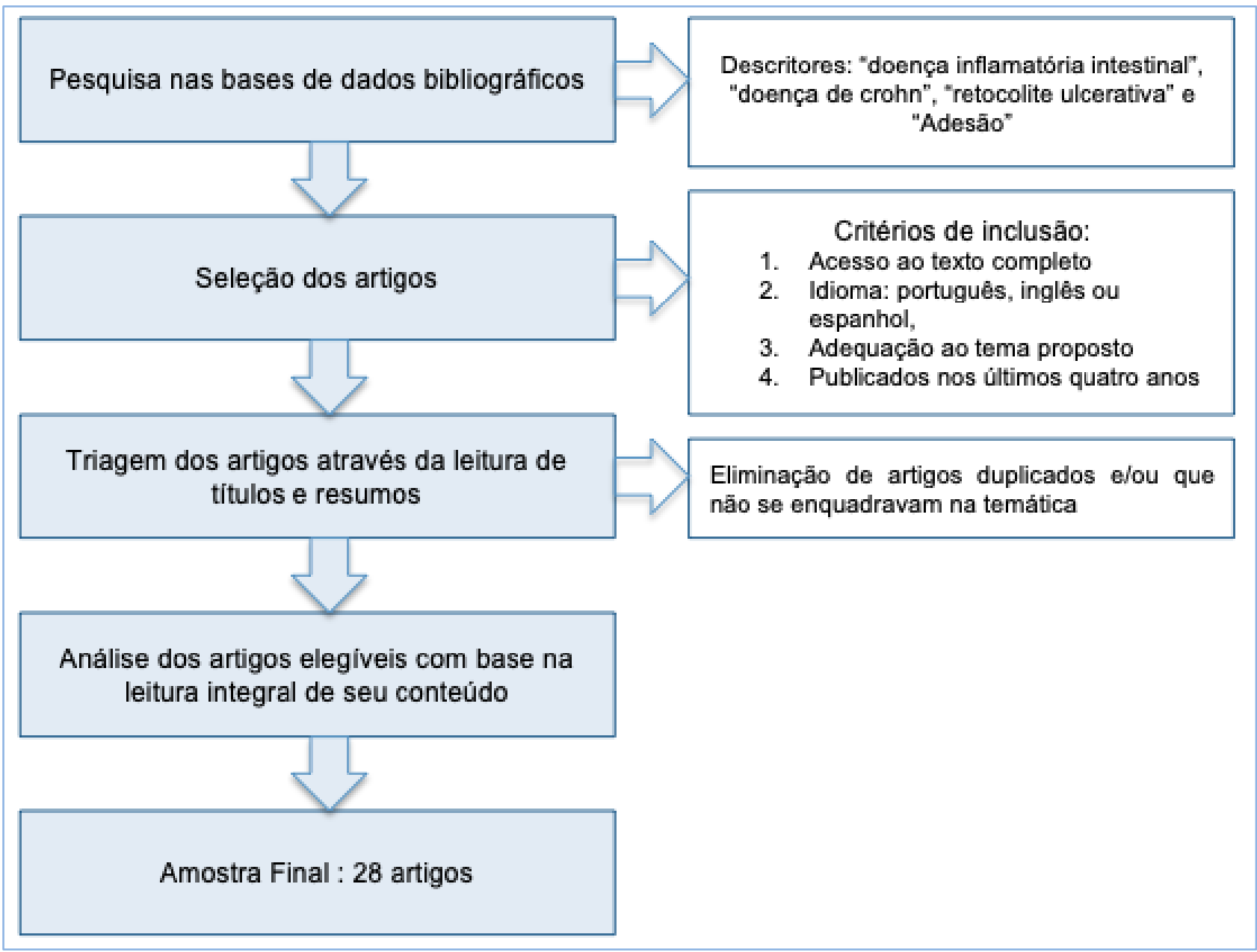

Fonte: Souza GF, et al., 2020.

\section{RESULTADOS E DISCUSSÃO}

Foram previamente selecionados 28 artigos e, a partir da análise crítica dos resumos, foram selecionados 20 artigos que atendiam aos critérios de inclusão propostos. A maioria das publicações se deu no ano de 2017. Pode-se observar também, que a maioria dos estudos utilizou métodos indiretos para avaliar a adesão ao tratamento medicamentoso na DII, conforme evidencia a Quadro 1. 
Quadro 1 - Descrição dos estudos selecionados.

\begin{tabular}{|c|c|c|}
\hline Título do artigo & Autor & Síntese dos estudos \\
\hline $\begin{array}{l}\text { Non-adherence to Anti-TNF Therapy is Associated with } \\
\text { Illness Perceptions and Clinical Outcomes in Outpatients } \\
\text { with Inflammatory Bowel Disease: Results from a } \\
\text { Prospective Multicentre Study }\end{array}$ & Van Der Have, et al. (2016). & $\begin{array}{l}\text { Estudo observacional multicêntrico. A adesão foi mensurada através da } \\
\text { escala MMAS- } 8 \text { e recargas de } 12 \text { meses em farmácias (posse de } \\
\text { medicamentos MPR). }\end{array}$ \\
\hline $\begin{array}{l}\text { Inflammatory Bowel Disease Pharmacist Adherence } \\
\text { Counseling Improves Medication Adherence in Crohn's } \\
\text { Disease and Ulcerative Colitis }\end{array}$ & Tiao DK, et al. (2017). & $\begin{array}{l}\text { Estudo prospectivo longitudinal multicêntrico sobre a influência do } \\
\text { aconselhamento farmacêutico na adesão aos medicamentos na } \\
\text { retocolite ulcerativa e doença de Crohn. Na avaliação da adesão foi } \\
\text { utilizado a Escala de Relatório de Adesão a Medicamentos (MARS) } \\
\text { juntamente com o questionário crenças sobre medicamento (BMQ). }\end{array}$ \\
\hline $\begin{array}{l}\text { Clinical Predictors of Future Nonadherence in } \\
\text { Inflammatory Bowel Disease }\end{array}$ & SEVERS M, et al. (2017). & $\begin{array}{l}\text { Estudo de coorte prospectivo multicêntrico com pacientes adultos com } \\
\text { doença de Crohn e retocolite ulcerativa. A adesão aos medicamentos foi } \\
\text { avaliada por meio de escala analógica ("EVA). }\end{array}$ \\
\hline $\begin{array}{l}\text { Re-phrasing the question: A simple tool for evaluation of } \\
\text { adherence to therapy in patients with inflammatory bowel } \\
\text { disease }\end{array}$ & ENGEL T, et al. (2017). & $\begin{array}{l}\text { Estudo transversal, com utilização de um em questionário, aplicado aos } \\
\text { pacientes com DIl tratados em um centro terciário. }\end{array}$ \\
\hline $\begin{array}{l}\text { Valoración de la adhesión terapéutica en la enfermedad } \\
\text { inflamatoria intestinal. Comparación entre una escala de } \\
\text { autoevaluación y un índice farmacéutico de dispensación } \\
\text { de medicamentos }\end{array}$ & DE CASTRO ML, et al. (2017). & $\begin{array}{l}\text { Estudo prospectivo sobre a avaliação da adesão terapêutica na DII. } \\
\text { Comparou uma escala de autoavaliação e um índice farmacêutico de } \\
\text { dispensação de medicamentos. }\end{array}$ \\
\hline $\begin{array}{l}\text { Current practice and clinicians' perception of medication } \\
\text { non-adherence in patients with inflammatory bowel } \\
\text { disease: A survey of } 98 \text { clinicians }\end{array}$ & SOOBRATY A, et al. (2017) & $\begin{array}{l}\text { O estudo desenvolveu uma pesquisa on-line para avaliar a percepção } \\
\text { de } 8 \text { especialistas em Dll sobre a adesão ao tratamento medicamentoso } \\
\text { em pacientes com DII. }\end{array}$ \\
\hline $\begin{array}{l}\text { Factors Predicting the Adherence to the Therapy of Italian } \\
\text { IBD Patients }\end{array}$ & BUCCI C, et al. (2017). & $\begin{array}{l}\text { Estudo prospectivo. Investigou a adesão à terapia e preditores de } \\
\text { adesão em portadores de DII. Avaliou adesão através da escala de } \\
\text { Morisky (MMAS-8). }\end{array}$ \\
\hline $\begin{array}{l}\text { Impact of a Patient Support Program on Patient Adherence } \\
\text { to Adalimumab and Direct Medical Costs in Crohn's } \\
\text { Disease, Ulcerative Colitis, Rheumatoid Arthritis, Psoriasis, } \\
\text { Psoriatic Arthritis, and Ankylosing Spondylitis }\end{array}$ & RUBIN DT, et al. (2017). & $\begin{array}{l}\text { Quantificou a relação entre a participação em um programa de suporte } \\
\text { ao paciente e os resultados de adesão em pacientes que iniciaram o } \\
\text { tratamento com adalimumabe. }\end{array}$ \\
\hline $\begin{array}{l}\text { Shared decision-making for biologic treatment of } \\
\text { autoimmune disease: influence on adherence, persistence, } \\
\text { satisfaction, and health care costs }\end{array}$ & LOFLAND JH, et al. (2017). & $\begin{array}{l}\text { Estudo transversal com adultos que iniciaram tratamento para doença } \\
\text { de Crohn ou colite ulcerosa ou artrite psoriática ou artrite reumatoide. } \\
\text { Utilizou como instrumento o Questionário Compartilhado de Tomada de } \\
\text { Decisão, Medida de Ativação do Paciente (PAM) e Escala de Adesão à } \\
\text { Medicação Morisky (MMAS). }\end{array}$ \\
\hline $\begin{array}{l}\text { Determining the degree of adherence to treatment in } \\
\text { inflammatory bowel disease patients }\end{array}$ & BALAII H, et al. (2018). & $\begin{array}{l}\text { Estudo prospectivo. Avaliou adesão ao tratamento medicamentoso em } \\
\text { pacientes com DII através do teste de Morisky (MMAS-8). Avaliou } \\
\text { também a qualidade de vida destes pacientes pelo Short-Form } 36 \text {. }\end{array}$ \\
\hline
\end{tabular}




\begin{tabular}{|c|c|c|}
\hline Título do artigo & Autor & $\begin{array}{l}\text { Síntese dos estudos } \\
\end{array}$ \\
\hline $\begin{array}{l}\text { Defining an Optimal Adherence Threshold for Patients } \\
\text { Taking Subcutaneous Anti-TNFs for Inflammatory Bowel } \\
\text { Diseases }\end{array}$ & GOVANI SM, et al. (2018). & $\begin{array}{l}\text { Estudo retrospectivo. Buscou definir um limiar ideal de adesão para } \\
\text { pacientes que tomam anti-TNFs subcutâneos para doenças } \\
\text { inflamatórias intestinais. }\end{array}$ \\
\hline $\begin{array}{l}\text { The adherence to the therapy in inflammatory bowel } \\
\text { disease: beyond the number of the tablets }\end{array}$ & RIBALDONE DG, et al. (2018). & $\begin{array}{l}\text { Estudo prospectivo cego sobre avaliação da adesão à mesalazina. A } \\
\text { adesão foi avaliada através do autorrelato dos pacientes. }\end{array}$ \\
\hline $\begin{array}{l}\text { Medication non-adherence in inflammatory bowel diseases } \\
\text { is associated with disability }\end{array}$ & PERRY J, et al. (2018). & $\begin{array}{l}\text { Estudo transversal não intervencionista. Investigou a associação entre a } \\
\text { não adesão à medicação e deficiência em portadores de DII. Utilizou os } \\
\text { seguintes instrumentos: Escala de Classificação de Adesão à Medicação } \\
\text { (MARS); Índice de Incapacidade Inflamatória Intestinal (IBD-DI) e } \\
\text { Crenças sobre Medicamentos (BMQ). }\end{array}$ \\
\hline $\begin{array}{l}\text { Higher education, professional occupation, and upper } \\
\text { socioeconomic status are associated with lower adherence } \\
\text { to medications in patients with inflammatory bowel disease }\end{array}$ & TOMAR SK, et al. (2019). & $\begin{array}{l}\text { Estudo observacional transversal. Avaliou taxas e preditores de adesão } \\
\text { à medicação em portadores de DIl. A adesão foi avaliada com um } \\
\text { questionário (baseado em entrevista) e definida como ingestão de } \\
\text { drogas }<80 \% \text { da dose prescrita. }\end{array}$ \\
\hline $\begin{array}{l}\text { Adherence to Asacol once daily versus divided regimen for } \\
\text { maintenance therapy in ulcerative colitis: a prospective, } \\
\text { multicenter, randomized study }\end{array}$ & PARK SK, et al. (2019). & $\begin{array}{l}\text { Estudo prospectivo, multicêntrico e randomizado. Foram incluídos } \\
\text { pacientes com RCU em remissão há mais de } 3 \text { meses. Para avaliar a } \\
\text { adesão, os pacientes inscritos mantiveram diários de medicamentos } \\
\text { (adesão autorreferida). }\end{array}$ \\
\hline $\begin{array}{l}\text { Retrospective Claims Analysis Indirectly Comparing } \\
\text { Medication Adherence and Persistence Between } \\
\text { Intravenous Biologics and Oral Small-Molecule Therapies } \\
\text { in Inflammatory Bowel Diseases }\end{array}$ & MORAN K, et al. (2019). & $\begin{array}{l}\text { Estudo de coorte retrospectiva. Foram incluídos pacientes adultos } \\
\text { diagnosticados com DII ou artrite reumatóide (AR) que iniciou o } \\
\text { tratamento com vedolizumabe, tofacitinibe ou infliximab. Identificou } \\
\text { diferenças na adesão e persistência com tratamentos com diferentes } \\
\text { vias de administração (intravenosa x oral) em DII. }\end{array}$ \\
\hline $\begin{array}{l}\text { Validación de Belief Medicines Questionnaire y Self- } \\
\text { efficacy for Appropriate Medication Use Scale para medir } \\
\text { adherencia al tratamiento farmacológico en pacientes com } \\
\text { enfermedad inflamatoria intestinal }\end{array}$ & BOZADA-GUTIÉRREZ KE, et al. (2019). & $\begin{array}{l}\text { Estudo prospectivo. Traduziu os instrumentos Questionário de } \\
\text { Medicamentos para Crenças (BMQ) e instrumentos de Autoeficácia para } \\
\text { Uso Apropriado de Medicamentos Scale (SEAMS), determinou sua } \\
\text { validade, confiabilidade e sensibilidade em pacientes mexicanos } \\
\text { diagnosticados com DII. }\end{array}$ \\
\hline $\begin{array}{l}\text { Factors associated with low adherence to oral } 5 \text { - } \\
\text { aminosalicylic acid in patients with ulcerative colitis }\end{array}$ & LEE J, et al. (2019). & $\begin{array}{l}\text { Estudo transversal multicêntrico em } 6 \text { hospitais universitários na Coréia. } \\
\text { A adesão à medicação foi avaliada usando o questionário Morisky } \\
\text { Medication Adherence Scale (MMAS-8). }\end{array}$ \\
\hline $\begin{array}{l}\text { Translation and initial validation of the Medication } \\
\text { Adherence Report Scale (MARS) in Italian patients with } \\
\text { Crohn's disease }\end{array}$ & SCRIBANO ML, et al. (2019). & $\begin{array}{l}\text { O objetivo do estudo foi analisar as propriedades psicométricas do } \\
\text { MARS-5 (Medication Adherence Report Scale) e identificar se a tradução } \\
\text { em italiano é adequada para avaliar a aderência a medicamentos em } \\
\text { pacientes italianos com doença de Crohn (CD). }\end{array}$ \\
\hline $\begin{array}{l}\text { The Telemedicine for Patients with Inflammatory Bowel } \\
\text { Disease (TELE-IBD) Clinical Trial: Qualitative Assessment } \\
\text { of Participants' Perceptions }\end{array}$ & QUINN CC, et al. (2019) & $\begin{array}{l}\text { O objetivo deste estudo, telemedicina para pacientes com DII } \\
\text { (TELE-IBD), foi comparar a atividade da doença e a qualidade de vida } \\
\text { (QV) em um ensaio clínico randomizado de } 1 \text { ano em pacientes com DII } \\
\text { recebendo telemedicina versus atendimento padrão. }\end{array}$ \\
\hline
\end{tabular}

Fonte: Souza GF et al., 2020. 
Após leitura crítica e sistematização dos dados, puderam-se evidenciar três grupos de discussão: adesão da farmacoterapia nas DII, preditores de não adesão ao tratamento e estratégias de intervenção para melhoraria da adesão aos medicamentos.

\section{Adesão da farmacoterapia nas DII}

De acordo com a literatura, a ocorrência de adesão ao tratamento medicamentoso em pacientes com doença inflamatória intestinal é heterogênea. Dos 20 trabalhos incluídos no estudo, 13 trabalhos avaliaram as taxas de adesão (VAN DER HAVE M, et al., 2016; BUCCI C, et al., 2017; DE CASTRO ML, et al., 2017; ENGEL T, et al., 2017; SEVERS M, et al., 2017; BALAIl H, et al., 2018; PERRY J, et al., 2018; RIBALDONE DG, et al., 2018; GOVANI SM, et al., 2018; BOZADA-GUTIÉRREZ KE, 2019; TOMAR SK, et al., 2019; LEE J, et al., 2019; PARK SK, et al., 2019). Todos estes trabalhos utilizaram métodos indiretos para avaliar adesão.

O método mais utilizado para avaliar a adesão foi a Escala de Adesão Terapêutica de Morisky de oito itens (MMAS-8) (VAN DER HAVE M, et al., 2016; BUCCI C, et al., 2017; BALAll H, et al., 2018; LEE J, et al., 2019). No entanto, houve grande variação entre os resultados encontrados, tendo havido segundo a escala, boa aderência, em apenas $12,4 \%$ dos pacientes no estudo de Balaii $\mathrm{H}$, et al. (2018) e de $71,5 \%$ no estudo de Bucci C, et al. (2017).

Essa grande variação de resultados mesmo com a utilização de mesmo método se deve a vários fatores, entre eles o tipo de estudo, a doença inflamatória intestinal estudada, a idade do paciente, a escolha da via de administração do medicamento, a população estudada, dentre outros (MORAN K, et al., 2019).

Um exemplo de variação ocorrida por alguns desses fatores descritos pode ser observado na comparação entre dois estudos multicêntrico que também usaram a escala MMAS-8. Van Der Have M, et al. (2019) avaliaram a adesão à terapia subcutânea com infliximabe e adalimumabe em 3 hospitais da Holanda e encontraram $24 \%$ dos pacientes com alta adesão, $48 \%$ com média adesão e apenas $28 \%$ de pacientes com baixa adesão. Já no estudo de Lee J, et al. (2019), que avaliaram a adesão ao 5-ASA (Ácido 5-aminosalicílico) em 6 hospitais na Coréia, mesmo sendo uma medicação de uso oral, em pacientes com colite ulcerosa, verificaram que $49,8 \%$ da população estudada apresentou baixa adesão.

Apenas 4 estudos utilizaram instrumentos combinados para analisar adesão. Um dos estudos utilizou a escala MMAS-8 em conjunto com a relação de posse de medicamentos (MPR) para investigar a adesão à terapia anti-TNF em pacientes ambulatoriais com DII. No estudo foi observado que $28 \%$ dos pacientes apresentaram MMAS- $8<6$, indicando baixa adesão e $25 \%$ dos pacientes apresentaram MPR $<80 \%$, indicando não adesão. Segundo os autores do estudo, embora a ferramenta MMAS-8 tenha se correlacionado com o comportamento do MPR, esta correlação foi baixa, sugerindo limitada função da MMAS-8 como ferramenta para prever adesão. No entanto, outros estudos que excluíram pacientes que receberam anti-TNF observaram associação significativa entre a ferramenta MMAS-8 e a MPR. Este fato pode ser explicado pelo fato da MMAS-8 ter sido originalmente desenvolvida para pacientes que utilizavam apenas medicação diária e várias perguntas não se aplicarem a medicações intravenosa, como por exemplo "Você cortou sua medicação sem informar seu médico?" (VAN DER HAVE M, et al., 2016; DE CASTRO ML, et al., 2017; BALAll H, et al., 2018; PERRY J, et al., 2018)

Outro estudo também evidenciou a limitação da MMAS-8. De Castro ML, et al. (2017) também utilizaram a MMAS-8 e a relação MRP para avaliar adesão terapêutica nas DII e identificaram, segundo a MMAS-8, baixa adesão em apenas $22 \%$ dos pacientes, enquanto a que a MRP mostrou que $37 \%$ dos pacientes apresentavam valor inadequado. Desse modo, o estudo demonstra que $15 \%$ de indivíduos com adesão inadequada foi classificado incorretamente usando o MMAS-8, tendo demonstrado baixa especificidade (34\%) e valor preditivo negativo (57\%). Esses resultados podem ser explicados pela tendência de haver superestimativa na avaliação da adesão quando o paciente é perguntado sobre ela, diminuindo assim a especificidade e aumentando o número de resultados falso-positivos desta ferramenta.

Perry J, et al. (2018) utilizou a associação de três ferramentas para avaliar adesão e estudou também a relação desta com a incapacidade do paciente. Utilizou como ferramentas: Escala de Adesão à Medicação (MARS), Brief Medication Questionaire (BMQ) e Índice de Incapacidade em pacientes com doença 
Inflamatória Intestinal (IBD-DI). Foi observado que $24 \%$ dos participantes do estudo não aderiram ao tratamento e a incapacidade, definida pela baixa capacidade funcional dos pacientes, correlacionou-se positivamente com a não adesão aos medicamentos $(r=0,38, P<0,0001)$. Os autores destacam que a MARS é uma ferramenta comumente usada que reflete o uso de vários medicamentos em vez de se limitar a uma classe de medicamentos. Ao contrário da MMAS-8, a MARS usa escalas Likert de 5 pontos para obter respostas dos sujeitos, evitando respostas dicotômicas "sim" ou "não", diminuindo a probabilidade de viés de resposta. Estudo de Scribano ML, et al. (2018) verificou se a tradução em italiano da MARS foi adequada para avaliar a adesão a medicamentos em pacientes italianos com doença de Crohn, o estudo confirmou a utilidade dessa ferramenta para esses pacientes.

Balaii $\mathrm{H}$, et al. (2018) também utilizou instrumentos combinados para analisar adesão na DII. No referido estudo foi utilizado a MMAS para detectar nível de adesão aos medicamentos, mas também a relação da adesão com a qualidade de vida, avaliada pelo questionário Short-Form 36 . Foi identificado que $46 \%$ dos pacientes apresentaram baixa adesão e não foi evidenciada relação entre nível de adesão e estado mental, físico ou qualidade de vida total.

Na presente revisão integrativa, além do que foi apresentado até o momento, foram evidenciados outros instrumentos para mensurar a adesão nas DII. Três trabalhos utilizaram questionários adaptados ao estudo, um estudo utilizou a escala visual analógica (EVA) e um avaliou adesão por meio do diário de medicamentos do paciente (ENGEL T, et al., 2017; RIBALDONE DG, et al., 2018; SEVERS M, et al., 2017; TOMAR SK, et al., 2019; PARK SK, et al., 2019). Na análise dos estudos, o que se observa é uma variedade de instrumentos utilizados para analisar adesão com critérios metodológicos diferenciados, o que dificulta a comparação entre os resultados encontrados.

\section{Preditores de não adesão ao tratamento nas DII}

Como apresentado nas análises dos estudos, observa-se a ocorrência de adesão inadequada em proporção considerável de pacientes com DII. Sabe-se que identificar fatores de risco para a não adesão ajuda a planejar intervenção para melhorar este fator, assim como os resultados clínicos em portadores de DII (BALAll H, et al., 2018). Dos 20 trabalhos incluídos no estudo, 7 abordaram preditores de não adesão ao tratamento nas DII (VAN DER HAVE M, et al., 2016; BUCCI C, et al., 2017; SEVERS M, et al., 2017; BALAll H, et al., 2018; PERRY J, et al., 2018; TOMAR SK, et al., 2019; LEE J, et al., 2019).

O estudo de Van Der Have M, et al. (2019) demonstrou que percepções negativas sobre como a doença afeta o bem-estar emocional do paciente e a percepção do mesmo frente à doença episódica aguda foram associadas à não adesão.

Preditores de não adesão relacionados à questão emocional do paciente também foram encontrados no estudo de Severs M, et al. (2017), no qual foi demonstrada associação da não adesão a crises da doença, sentimentos de ansiedade ou depressão, além da relação também desses fatores com menor idade ao diagnóstico. Este estudo também verificou que em pacientes com DC, um melhor controle sobre a doença e uma adesão nos 6 primeiros meses de acompanhamento foram associadas com menor risco de comportamento não aderente. Do mesmo modo, em pacientes com RCU, foi observado que fatores como maior nível de entendimento da doença e maior influência da doença no humor dos pacientes foram associados a menor risco de comportamento não aderente.

No entanto, diferentemente dos dois últimos estudos que foram apresentados nesta revisão, Balaii $\mathrm{H}$, et al. (2018) não encontrou relação entre nível de adesão e estado mental ou qualidade de vida total. A não adesão ao tratamento na DII pelos primeiros 6 meses de acompanhamento, citada como preditor de não adesão futura por Severs $M$, et al. (2017) também se configurou um preditor de não adesão ao tratamento nos estudos de Tomar SK, et al. (2019).

De acordo com este estudo, entre as razões predominantes para a não adesão ao tratamento em pacientes com DII está o esquecimento de doses do medicamento, o que se não orientado no início, aumenta a falha durante o tratamento futuro. Além deste, outros preditores evidenciados foram indisponibilidade de medicamentos, sentir-se melhor, efeitos adversos e custo do tratamento, enquanto educação dos pacientes, 
ocupação e status socioeconômico mostraram associação negativa com o nível de adesão. Diferente do referido por outros estudos realizados com pacientes de outras doenças, os pacientes com status socioeconômico elevado, com grau de escolaridade alto foram os menos aderentes (47\%), enquanto os pacientes dos estratos socioeconômicos inferiores, analfabetos e desempregados tiveram a maior adesão (100\%) (TOMAR SK, et al., 2019).

Estudos também avaliaram a idade dos pacientes como um fator preditor de não adesão. Dos trabalhos analisados na presente revisão, 2 encontraram menor idade como preditor de não adesão nas DII. De acordo com estes estudos, pacientes mais jovens tendem a ser menos aderentes à terapia, estando entre os principais motivos relatados pelos pacientes "incômodo de aderir ao plano de medicamentos" e "eles se sentem melhor" (BUCCI C, et al., 2017; SEVERS M, et al., 2017).

Outros fatores como etilismo e tabagismo também foram associados à não adesão ao tratamento na DII. Um estudo multicêntrico em 6 hospitais na Coréia analisou fatores associados à baixa adesão ao uso oral do Ácido 5-aminosalicílico em pacientes com RCU. Observou-se que, idade $<40$ anos, consumo de álcool e tabagismo atual foram associados à baixa adesão ao 5-ASA oral, embora os dois últimos preditores só se relacionaram com não adesão nos homens (LEE J, et al., 2019).

Perry J, et al. (2018) observou que a não adesão à medicação foi associado a um aumento da incapacidade na DII. Sexo feminino, maior gravidade da doença e preocupações com medicamentos foram preditores adicionais de incapacidade.

Nesse contexto, os preditores podem ser ferramentas importantes para a identificação de não adesão precoce ou futura, possibilitando intervenções individualizadas para a melhora da adesão e, com isso, prevenindo um curso desvantajoso da doença a longo prazo (SEVERS M, et al., 2017). Os principais preditores de não adesão ao tratamento medicamentoso em portadores de DII está descrito no Quadro 2.

Quadro 2 - Principais preditores de não adesão ao tratamento farmacológico em portadores de DII encontrados no estudo.

\begin{tabular}{|c|c|}
\hline Preditores & Autores \\
\hline Percepções negativas sobre como a doença & Van Der Have M, et al. (2019) \\
\hline Ansiedade ou depressão & Severs M, et al. (2017) \\
\hline Menor idade ao diagnóstico & Severs M, et al. (2017). Bucci C, et al (2017) \\
\hline Esquecimento de doses do medicamento & Tomar SK, et al. (2019) \\
\hline Indisponibilidade de medicamentos & Tomar SK, et al. (2019) \\
\hline Sentir-se melhor & Tomar SK, et al. (2019) \\
\hline Efeitos adversos & Tomar SK, et al. (2019) \\
\hline Custo do tratamento & Tomar SK, et al. (2019) \\
\hline Status socioeconômico elevado & Tomar SK, et al. (2019) \\
\hline Alto grau de escolaridade & Tomar SK, et al. (2019) \\
\hline Etilismo e tabagismo & Lee J, et al. 2019 \\
\hline
\end{tabular}

Fonte: Souza GF, et al., 2020.

\section{Estratégias de intervenção para melhorar a adesão aos medicamentos}

Dentre os 20 estudos analisados, 6 discutem formas de melhorar a adesão ao tratamento medicamentoso (SEVERS M, et al., 2017; BALAIl H, et al., 2018; RUBIN DT, et al., 2017; SOOBRATY A, et al., 2017; TIAO DK, et al., 2017; QUINN CC, et al., 2019).

Em estudo longitudinal que avaliou preditores de futura não adesão ao tratamento medicamentoso em pacientes com DII observou que maior compreensão da doença estava associado à adesão ao tratamento medicamentoso, assim como com percepções mais fortes da necessidade de tratamento e menos preocupações sobre aspectos negativos do tratamento (SEVERS M, et al., 2017).

De acordo com os autores do estudo, esses achados corroboram o modelo do senso comum, no qual é declarado que as crenças dos pacientes desempenham papel importante na modificação da doença. Os autores enfatizam também, que esses achados abrem novos caminhos para intervenções terapêuticas, sendo 
importante discutir percepções sobre a doença e o tratamento relacionado a todos os pacientes com DII, especialmente se houver suspeita de não adesão (SEVERS M, et al., 2017).

Outros trabalhos sugeriram que maior atuação dos profissionais de saúde pode contribuir no tratamento dos pacientes com DII (RUBIN DT, et al., 2017; SOOBRATY A, et al., 2017; LOFLAND JH, et al., 2017). Segundo Balaii $\mathrm{H}$, et al. (2018), um tratamento bem-sucedido é acompanhado de boa cooperação entre médico e paciente, a fim de alcançar a melhor maneira de controlar a doença.

Além do médico, outros profissionais da saúde também podem colaborar na adesão aos medicamentos na DII, entre eles farmacêuticos e enfermeiros. Um dos trabalhos analisados na presente revisão verificou que o aconselhamento farmacêutico contribuiu na diminuição da não adesão à medicação em portadores de DII. O aconselhamento personalizado sobre adesão ao tratamento prestado pelo farmacêutico, demonstrou ser ferramenta importante para aumentar a adesão à farmacoterapia na DII (TIAO DK, et al., 2017).

Outro trabalho analisado propôs a telemedicina como ferramenta que pode contribuir para maior adesão ao tratamento nas DII. Por meio do sistema TELE-IBD (telemedicina para pacientes com doença inflamatória intestinal), o monitoramento remoto foi realizado via mensagens de texto SMS (serviço de mensagens curtas) (QUINN CC, et al., 2019). Nesse estudo a telemedicina teve boa aceitação em pacientes com DII, além dos pacientes terem relatado melhora na compreensão da doença, monitorando seus sintomas e sentindo-se conectado ao seu médico.

Balaii $\mathrm{H}$, et al. (2018) destacam que avaliação regular da adesão ao medicamento seria benéfico para o acompanhamento e propõem a avaliação de um sistema abrangente com perguntas mais precisas para medir a adesão entre pacientes com DII.

Como visto, embora sete dos trabalhos analisados tenham enfocado a importância de estratégias de intervenção para melhora de adesão, apenas dois testaram estratégias nos pacientes com DII (TIAO DK, et al., 2017; QUINN CC, et al., 2019), indicando a necessidade de mais estudos com este enfoque nos referidos pacientes.

\section{CONSIDERAÇÕES FINAIS}

Houve diferença no percentual de adesão ao tratamento medicamentoso em pacientes com DII de acordo com as particularidades dos instrumentos de avaliação utilizados nos referidos estudos e população analisada. No entanto, independente dos métodos utilizados nos estudos, se observa ocorrência relevante de não adesão ao tratamento nas DII. Entre os preditores de não adesão ao tratamento farmacológico em portadores de DII, destaca-se a percepção negativa do paciente em relação a doença, sentimentos de ansiedade e depressão, menor idade ao diagnóstico, esquecimento de doses do medicamento, indisponibilidade de medicamentos, dentre outros. Somente dois estudos testaram estratégias direcionadas a melhorar a adesão em indivíduos com DII, indicando a necessidade de mais estudos com este enfoque.

\section{REFERÊNCIAS}

1. BALAll $\mathrm{H}$, et al. Determining the degree of adherence to treatment in inflammatory bowel disease patients. Gastroenterol Hepatol Bed Bench. 2018; 11(1): 39-44.

2. BARBOSA JPC. Avaliação da anemia dos pacientes com doença inflamatória intestinal. Trabalho de Conclusão de Residência Médica (Residência Médica em Gastroenterologia) - Hospital Geral de Fortaleza, Fortaleza, 2018.

3. BAUMGART DC, SANDBORN WJ. Crohn's disease. Lancet. 2012; 380:1590-1605.

4. BOZADA-GUTIÉRREZ KE. Validación de Belief Medicines Questionnaire y Self-efficacy for Appropriate Medication Use Scale para medir adherencia al tratamiento farmacológico en pacientes con enfermedad inflamatoria intestinal. Gaceta Medica de Mexico. 2019; 155(2): 124-129.

5. BUCCI C, et al. Factors predicting the adherence to the therapy of Italian IBD patients. Gastroenterology Research and Practice. 2017; 1-6.

6. CARDOZO WS, SOBRADO CW. Doença Inflamatória Intestinal. 2nd ed. Barueri-SP: Editora Manole, 2015; 772p.

7. CONRAD K, et al. Diagnosis and classification of ulcerative colitis. Autoimmunity Reviews. 2014; 13(4): 463-466.

8. DE CASTRO ML, et al. Valoración de la adhesión terapéutica en la enfermedad inflamatoria intestinal. Comparación entre una escala de autoevaluación y un índice farmacéutico de dispensación de medicamentos. Revista Española de Enfermedades Digestivas. 2017; 109(8): 542-551.

9. ENGEL T, et al. Re-phrasing the question: a simple tool for evaluation of adherence to therapy in patients with inflammatory bowel disease. United European Gastroenterology Journal, 2017; 5(6): 880-886. 
10. GASPARINI RG. Incidência e prevalência de doenças inflamatórias intestinais no estado de São Paulo - Brasil. Tese (Doutorado em Bases gerais da cirurgia). Faculdade de Medicina de Botucatu, Universidade Estadual Paulista. Botucatu, 2018; $91 \mathrm{p}$.

11. GLOMBIEWSKI JA, et al. Medication adherence in the general population. PLoS One, San Francisco. 2012; 7(2): 50537.

12. GOVANI SM, et al. Defining an optimal adherence threshold for patients taking subcutaneous anti-TNFs for inflammatory bowel diseases. The American Journal of Gastroenterology. 2018; 113(2): 276-282.

13. HANAUER SB, PRESENT DH. The state of the art in the management of inflammatory bowel disease. Reviews in gastroenterological disorders. 2003; 3: 81-92.

14. LEDDIN D, et al. Decreasing incidence of inflammatory bowel disease in eastern Canada: a population database study. BMC gastroenterology. 2014; 14(1): 140.

15. LEE J, et al. Factors associated with low adherence to oral 5-aminosalicylic acid in patients with ulcerative colitis. PLoS ONE. 2019; 14(3): 1-9.

16. LOFLAND JH, et al. Shared decision-making for biologic treatment of autoimmune disease: influence on adherence, persistence, satisfaction, and health care costs. Patient Prefer Adherence. 2017; 11: 947-958.

17. LOGAN I, BOWLUS CL. The geoepidemiology of autoimmune intestinal diseases. Autoimmunity reviews. 2010; 9(5): 372-378.

18. MARANHÃO DDA, et al. Características e diagnóstico diferencial das doenças inflamatórias intestinais. Jornal brasileiro de medicina. 2015; 103(1): 9-15.

19. MARINKER M, SHAW J. Not to be taken as directed: putting concordance for taking medicines into practice. British Medical Journal, 2003; 326(7385): 348-349.

20. MISZPUTEN SJ. Terapêutica não biológica das doenças inflamatórias intestinais. In: Cury DB, Moss AC. Doenças inflamatórias intestinais: retocolite ulcerativa e doença de Crohn. Rio de Janeiro: Editora Rubio, 2011; 464p.

21. MOLODECKY NA, et al. Increasing incidence and prevalence of the inflammatory bowel diseases with time, based on systematic review. Gastroenterology, 2012; 142(1): 46-54.

22. MORAN K, et al. Retrospective claims analysis indirectly comparing medication adherence and persistence between intravenous biologics and oral small-molecule therapies in inflammatory bowel diseases. Advances in Therapy. 2019; 36(9): 2260-2272.

23. MOREIRA AL, et al. IBD Epidemiology: What is Going on in the Developing World? Results from 163,000 Patients. Gastroenterology. 2019; 156(6): 73.

24. OBRELI-NETO PR, et al. Métodos de avaliação de adesão à farmacoterapia. Revista Brasileira de Farmácia, 2012; 93(4): 403-10.

25. PARENTE JM, et al. Inflammatory bowel disease in an underdeveloped region of Northeastern Brazil. World Journal of Gastroenterology. 2015; 28(4): 1197-206.

26. PARK SK, et al. Adherence to Asacol once daily versus divided regimen for maintenance therapy in ulcerative colitis: a prospective, multicenter, randomized study. Intestinal Research, 2019; 17(3): 349-356.

27. PERRY J, et al. Medication non-adherence in inflammatory bowel diseases is associated with disability. Intestinal Research. 2018; 16(4): 571-578.

28. QUINN CC, et al. The telemedicine for patients with inflammatory bowel disease (Tele-IBD) clinical trial: qualitative assessment of participants' perceptions. Journal of Medical Internet Research. 2019; 21(6): e14165.

29. RIBALDONE DG, et al. The adherence to the therapy in inflammatory bowel disease: beyond the number of the tablets. Scandinavian Journal of Gastroenterology. 2018; 53(2): 141-146.

30. RUBIN DT, et al. Impact of a patient support program on patient adherence to adalimumab and direct medical costs in Crohn's disease, ulcerative colitis, rheumatoid arthritis, psoriasis, psoriatic arthritis, and ankylosing spondylitis. Journal of Managed Care \& Specialty Pharmacy. 2017; 23(8): 859-867.

31. SCRIBANO ML, et al. Translation and initial validation of the Medication Adherence Report Scale (MARS) in Italian patients with Crohn's Disease. Digestive and Liver Disease. 2018; 51(5): 640-647.

32. SEVERS M, et al. Clinical Predictors of Future Nonadherence in Inflammatory Bowel Disease. Inflammatory Bowel Diseases. 2017; 23(9): 1568-1576.

33. SILVA BC, et al. The Demographic and Clinical Characteristics of Ulcerative Colitis in a Northeast Brazilian Population. BioMed Research International, 2015.

34. SOOBRATY A, et al. Current practice and clinicians' perception of medication non-adherence in patients with inflammatory bowel disease: a survey of 98 clinicians. World Journal of Gastrointestinal Pharmacology and Therapy, 2017; 8: 67-73.

35. SOUZA ACG, et al. Retocolite Ulcerativa associada à Doença de Crohn: um estudo de caso. Salvador, Escola Bahiana de Medicina e Saúde Pública, 2015.

36. SOUZA TT. Morbidade e mortalidade relacionadas a medicamentos: revisão sistemática e meta-análise de estudos observacionais, PR. Dissertação (Mestrado em Ciências Farmacêuticas) - Programa de Pós-Graduação em Ciências Farmacêuticas, Setor de Ciências da Saúde, Universidade Federal do Paraná, Curitiba, 2013; 326 p.

37. TIAO DK, et al. Inflammatory bowel disease pharmacist adherence counseling improves medication adherence in Crohn's disease and ulcerative colitis. Inflammatory Bowel Diseases. 2017; 23(8): 1257-1261.

38. TOMAR SK, et al. Higher education, professional occupation, and upper socioeconomic status are associated with lower adherence to medications in patients with inflammatory bowel disease. Journal of Gastroenterology and Hepatology Open. 2019; 3(4): 302-309.

39. VAN DER HAVE M, et al. Non-adherence to Anti-TNF Therapy is Associated with Illness Perceptions and Clinical Outcomes in Outpatients with Inflammatory Bowel Disease: Results from a Prospective Multicentre Study. Journal of Crohn's and Colitis, 2016; 10(5): 549-555.

40. WORLD HEALTH ORGANIZATION. 2003. Adherence to long-term therapies: evidence for action. Disponível em: <http://www.who.int/chronic_conditions/en/adherence_report.pdf>. Acesso em: 20 ago. 2020. 